\title{
Asymptotic behavior of the number of solutions for non-Archimedean Diophantine approximations
}

by

Hitoshi Nakada (Yokohama) and Rie Natsui (Yokohama and Tokyo)

1. Introduction. Let $\mathbb{F}_{q}$ be a finite field with $q$ elements, $\mathbb{F}_{q}[X]$ the set of polynomials with $\mathbb{F}_{q}$-coefficients, $\mathbb{F}_{q}(X)$ the set of rational functions with $\mathbb{F}_{q}$-coefficients, and $\mathbb{F}_{q}\left(\left(X^{-1}\right)\right)$ the set of formal Laurent series with $\mathbb{F}_{q^{-}}$-coefficients. For an element $f=a_{n} X^{n}+a_{n-1} X^{n-1}+\cdots \in \mathbb{F}_{q}\left(\left(X^{-1}\right)\right)$ with $a_{i} \in \mathbb{F}_{q}, a_{n} \neq 0$, we define

$$
\operatorname{deg} f=n \quad \text { and } \quad|f|=q^{\operatorname{deg} f} .
$$

In particular, we put $\operatorname{deg} 0=-\infty$ and $|0|=0$ for $0 \in \mathbb{F}_{q}$. Moreover define

$$
\mathbb{L}=\left\{f \in \mathbb{F}_{q}\left(\left(X^{-1}\right)\right): \operatorname{deg} f<0\right\} .
$$

We consider the topology on $\mathbb{L}$ induced by the metric $d(f, g)=|f-g|$ for $f, g \in \mathbb{L}$, and denote by $\mu$ the Haar probability measure on $\mathbb{L}$. K. Inoue and H. Nakada [2] showed that for any sequence $\left(l_{n}: n \geq 1\right)$ of non-negative integers the inequality

$$
\left|f-\frac{P}{Q}\right|<\frac{1}{q^{2 n+l_{n}}}, \quad P, Q \in \mathbb{F}_{q}[X], \operatorname{deg} Q=n, \text { and } P, Q \text { co-prime, }
$$

has infinitely many solutions $P / Q \in \mathbb{F}_{q}(X)$ for $\mu$-a.e. $f$ if and only if

$$
\sum_{n=1}^{\infty} \frac{1}{q^{l_{n}}}=\infty
$$

Moreover, the proof implied that the law of large numbers holds in the following sense:

$\sharp\left\{\frac{P}{Q}:(1)\right.$ holds and $\left.1 \leq n \leq N\right\}=\frac{q-1}{q} \Phi(N)+O\left(\Phi(N)^{1 / 2} \log ^{3 / 2+\varepsilon} \Phi(N)\right)$ for $\mu$-a.e. $f$, where

$$
\Phi(N)=\sum_{n=1}^{N} \frac{1}{q^{l_{n}}} .
$$

2000 Mathematics Subject Classification: 11J61, 11J83, 11K60. 
Indeed, this is a consequence of the quantitative Borel-Cantelli lemma by W. Philipp [5]. In the following, we write $(P, Q)=1$ if $P$ and $Q$ are co-prime.

In this paper, we consider the same inequality without the co-primality condition. Then there are two different types of inequalities, which are equivalent but involve different ideas:

$$
\begin{aligned}
& \text { (I) }\left|f-\frac{P}{Q}\right|<\frac{1}{q^{2 n+l_{n}}}, \quad \operatorname{deg} Q=n, \\
& \text { (II) }|Q f-P|<\frac{1}{q^{n+l_{n}}}, \quad \operatorname{deg} Q=n .
\end{aligned}
$$

For (I), "solutions" are rational functions, while for (II), there are pairs of polynomials $Q$ and $P$. When we consider the problem of approximation of Laurent power series by rational functions, we should look at the first inequality. However, it would be rather natural to consider (II) when we discuss properties of generalized Kronecker sequences (see [3] and [4]). Indeed, the Kronecker sequence of $f$ is the sequence consisting of the negative degree part of $Q f$ for $Q \in \mathbb{F}_{q}[X]$ and it is of the form $Q f-P$. In $\S 2$ we will show the strong law of large numbers for solutions of the inequality (I). We cannot apply the quantitative Borel-Cantelli lemma for this result since some events associated to (I) have strong dependence. However, we can show that the probability of such events is small enough and we can apply the Basic Lemma stated below. This lemma is an abstract version of the quantitative Borel-Cantelli lemma and has been used in the metric theory of the classical Diophantine approximations, the real numbers case (see Sprindžuk [6]). In $\S 3$, we also consider the strong law of large numbers for solutions of (II). We use the non-Archimedean continued fractions to prove this, since we cannot make use of the Basic Lemma. For this reason, it is not possible to estimate the remainder term in this case. Actually, in $\S 2$ and $\S 3$, we show the following. For $n \geq 0$ we put

$$
\begin{gathered}
W_{n}=\left\{\frac{P}{Q}: P, Q \in \mathbb{F}_{q}[X], \operatorname{deg} Q=n, \operatorname{deg} P<n,(P, Q)=1\right\}, \\
W_{n}^{*}=\left\{\frac{P}{Q}: P, Q \in \mathbb{F}_{q}[X], \operatorname{deg} Q=n, \operatorname{deg} P<n\right\}, \\
W_{n}^{* *}=\left\{(P, Q): P, Q \in \mathbb{F}_{q}[X], \operatorname{deg} Q=n, \operatorname{deg} P<n\right\} . \\
\text { Main Results. }
\end{gathered}
$$

(i) (Theorem 1) For any sequence $\left(l_{n}: n \geq 1\right)$ of non-negative integers, we have

$$
\begin{aligned}
\sharp\left\{\frac{P}{Q} \in W_{n}^{*}\right. & \left.: 1 \leq n \leq N,\left|f-\frac{P}{Q}\right|<\frac{1}{q^{2 n+l_{n}}}\right\} \\
& =\frac{q}{q+1} \Phi(N)+O\left(\Phi(N)^{1 / 2} \log ^{3 / 2+\varepsilon} \Phi(N)\right) \quad \text { for } \mu \text {-a.e. } f .
\end{aligned}
$$


(ii) (Theorem 3) If $\left(l_{n}: n \geq 1\right)$ satisfies

(A1) $\left(l_{n}: n \geq 1\right)$ is non-decreasing and $\sum_{n=1}^{\infty} 1 / q^{l_{n}}=\infty$,

(A2) there exists a constant $D>1$ such that $j_{k+1} \geq D j_{k}$ for $k \geq 1$, where

$$
\begin{aligned}
& j_{1}:=\min \left\{n \geq 2: l_{n}-l_{n-1}>0\right\}, \\
& j_{k}:=\min \left\{n>j_{k-1}: l_{n}-l_{n-1}>0\right\} \quad \text { for } k \geq 2,
\end{aligned}
$$

then

$$
\begin{aligned}
\lim _{N \rightarrow \infty} \frac{\sharp\left\{(P, Q) \in W_{n}^{* *}: 1 \leq n \leq N,|f-P / Q|<1 / q^{2 n+l_{n}}\right\}}{\Phi(N)}=q-1 & \\
& \text { for } \mu \text {-a.e. } f .
\end{aligned}
$$

As mentioned before, the following is essential in $\S 2$.

BASic Lemma (Sprindžuk [6, p. 45]). Let $\left(\xi_{n}(\omega): n \geq 1\right)$ be a sequence of random variables defined on a probability space $(\Omega, \mathcal{B}, P)$. Moreover let $\left(\eta_{n}: n \geq 1\right)$ and $\left(\widehat{\eta}_{n}: n \geq 1\right)$ be sequences of real numbers such that

$$
0 \leq \eta_{n} \leq \widehat{\eta}_{n} \leq 1 \quad \text { for } n \geq 1 \text {. }
$$

If

$$
\int_{\Omega}\left(\sum_{n=N_{1}}^{N_{2}} \xi_{n}(\omega)-\eta_{n}\right)^{2} d P \ll \sum_{n=N_{1}}^{N_{2}} \widehat{\eta}_{n}
$$

for any positive integers $N_{1}<N_{2}$, then

$$
\sum_{n=1}^{N} \xi_{n}(\omega)=\sum_{n=1}^{N} \eta_{n}+O\left(\Psi(N)^{1 / 2} \log ^{3 / 2+\varepsilon} \Psi(N)\right) \quad \text { for P-a.e. } \omega,
$$

where $\varepsilon>0$ is arbitrary and $\Psi(N)=\sum_{n=1}^{N} \widehat{\eta}_{n}$.

Throughout this paper, $A(n) \sim B(n)$ means $\lim _{n \rightarrow \infty} B(n) / A(n)=1$, and $A(n) \ll B(n)$ means there exists a constant $c>0$ such that $A(n)<$ $c B(n)$ for any $n \geq 1$.

2. The number of solutions as rational functions. Let $\left(l_{n}: n \geq 1\right)$ be a sequence of non-negative integers. In this section, we consider the following inequality:

$$
\left|f-\frac{P}{Q}\right|<\frac{1}{q^{2 n+l_{n}}}, \quad \frac{P}{Q} \in W_{n}^{*},
$$

for $f \in \mathbb{L}$. We define

$$
F_{n}^{*}=\left\{f \in \mathbb{L}: \text { there exists } \frac{P}{Q} \in W_{n}^{*} \text { such that }\left|f-\frac{P}{Q}\right|<\frac{1}{q^{2 n+l_{n}}}\right\}
$$


and

$$
F_{n, k}^{*}=\left\{f \in \mathbb{L}: \text { there exists } \frac{P}{Q} \in W_{n-k} \text { such that }\left|f-\frac{P}{Q}\right|<\frac{1}{q^{2 n+l_{n}}}\right\}
$$

for $0 \leq k \leq n$, where we note that $W_{0}=\{0\}$.

LEMMA 1.

$$
F_{n}^{*}=\bigcup_{k=0}^{n} F_{n, k}^{*} \quad \text { (disjoint union). }
$$

Proof. It is clear that $f \in F_{n}^{*}$ when $f \in F_{n, k}^{*}$ for some $0 \leq k \leq n$. Suppose that $f \in F_{n}^{*}$ and there are $P_{1} / Q_{1}, P_{2} / Q_{2} \in W_{n}^{*}$ such that

$$
\left|f-\frac{P_{i}}{Q_{i}}\right|<\frac{1}{q^{2 n+l_{n}}}, \quad i=1,2 .
$$

Then

$$
\left|\frac{P_{1}}{Q_{1}}-\frac{P_{2}}{Q_{2}}\right|<\frac{1}{q^{2 n+l_{n}}} .
$$

Since $\operatorname{deg} Q_{1}=\operatorname{deg} Q_{2}=n$, we have $\left|P_{1} / Q_{1}-P_{2} / Q_{2}\right| \geq 1 / q^{2 n}$ unless $P_{1} / Q_{1}=P_{2} / Q_{2}$. Since every $P / Q \in F_{n}^{*}$ belongs to only one $F_{n, k}^{*}$ for $1 \leq$ $k \leq n$, we have the assertion of this lemma.

LEMma 2.

$$
\sharp W_{n}^{*}=\frac{q}{q+1} q^{2 n}+\frac{1}{q+1} .
$$

Proof. It is obvious that $\sharp W_{n}^{*}=\sum_{k=0}^{n} \sharp W_{k}$. Since $\sharp W_{k}=q^{2 k}-q^{2 k-1}$ for $k \geq 1$ (see [2]), and $\sharp W_{0}=1$, we have the assertion.

Theorem 1. We have

$$
\begin{aligned}
\sharp\left\{\frac{P}{Q} \in W_{n}^{*}\right. & \left.: 1 \leq n \leq N,\left|f-\frac{P}{Q}\right|<\frac{1}{q^{2 n+l_{n}}}\right\} \\
& =\frac{q}{q+1} \Phi(N)+O\left(\Phi(N)^{1 / 2} \log ^{3 / 2+\varepsilon} \Phi(N)\right) \quad \text { for } \mu \text {-a.e. } f .
\end{aligned}
$$

Proof. It is sufficient to show that

$$
\sharp\left\{1 \leq n \leq N: f \in F_{n}^{*}\right\}=\frac{q}{q+1} \Phi(N)+O\left(\Phi(N)^{1 / 2} \log ^{3 / 2+\varepsilon} \Phi(N)\right)
$$

for $\mu$-a.e. $f$.

For this, we first show that $\sharp\left\{1 \leq n \leq N: f \in F_{n}^{*}\right\}=\widetilde{\Phi}(N)+O\left(\widetilde{\Phi}(N)^{1 / 2} \log ^{3 / 2+\varepsilon} \widetilde{\Phi}(N)\right) \quad$ for $\mu$-a.e. $f$ with $\widetilde{\Phi}(N)=\sum_{n=1}^{N} \mu\left(F_{n}^{*}\right)$. We put

$$
\xi_{n}(\cdot)=\chi_{F_{n}^{*}}(f) \text { and } \eta_{n}=\widehat{\eta}_{n}=\mu\left(F_{n}^{*}\right)
$$


and apply the Basic Lemma. To do this, it is enough to show that

$$
\sum_{n=N_{1}}^{N_{2}} \sum_{n^{\prime}=N_{1}}^{N_{2}}\left(\mu\left(F_{n}^{*} \cap F_{n^{\prime}}^{*}\right)-\mu\left(F_{n}^{*}\right) \mu\left(F_{n^{\prime}}^{*}\right)\right) \ll \sum_{n=N_{1}}^{N_{2}} \mu\left(F_{n}^{*}\right) .
$$

From Lemma 1, we see that

$$
\begin{aligned}
\mu\left(F_{n}^{*} \cap F_{n^{\prime}}^{*}\right)-\mu\left(F_{n}^{*}\right) & \mu\left(F_{n^{\prime}}^{*}\right) \\
& =\sum_{k=0}^{n} \sum_{k^{\prime}=0}^{n^{\prime}}\left(\mu\left(F_{n, k}^{*} \cap F_{n^{\prime}, k^{\prime}}^{*}\right)-\mu\left(F_{n, k}^{*}\right) \mu\left(F_{n^{\prime}, k^{\prime}}^{*}\right)\right) .
\end{aligned}
$$

From [2] we infer that

$$
\mu\left(F_{n, k}^{*} \cap F_{n^{\prime}, k^{\prime}}^{*}\right)=0 \text { or } \mu\left(F_{n, k}^{*}\right) \mu\left(F_{n^{\prime}, k^{\prime}}^{*}\right)
$$

whenever $n-k \neq n^{\prime}-k^{\prime}$. Thus we have

$$
\begin{aligned}
\operatorname{RHS}(4) & \leq \sum_{k=n-n^{\prime}}^{n}\left(\mu\left(F_{n, k}^{*} \cap F_{n^{\prime}, n^{\prime}+k-n}^{*}\right)-\mu\left(F_{n, k}^{*}\right) \mu\left(F_{n^{\prime}, n^{\prime}+k-n}^{*}\right)\right) \\
& \leq \sum_{k=n-n^{\prime}}^{n} \mu\left(F_{n, k}^{*} \cap F_{n^{\prime}, n^{\prime}+k-n}^{*}\right) \quad \text { for } n<n^{\prime} .
\end{aligned}
$$

Now

$$
\begin{aligned}
& \sum_{n=N_{1}}^{N_{2}} \sum_{n^{\prime}=N_{1}}^{N_{2}}\left(\mu\left(F_{n}^{*} \cap F_{n^{\prime}}^{*}\right)-\mu\left(F_{n}^{*}\right) \mu\left(F_{n^{\prime}}^{*}\right)\right) \\
& \quad \leq \sum_{n=N_{1}}^{N_{2}} \mu\left(F_{n}^{*}\right)+2\left\{\sum_{n=N_{1}}^{N_{2}} \sum_{n^{\prime}=N_{1}}^{n-1} \mu\left(F_{n}^{*} \cap F_{n^{\prime}}^{*}\right)-\mu\left(F_{n}^{*}\right) \mu\left(F_{n^{\prime}}^{*}\right)\right\} .
\end{aligned}
$$

From (5), we find that

$$
\begin{aligned}
& \sum_{n=N_{1}}^{N_{2}} \sum_{n^{\prime}=N_{1}}^{n-1}\left(\mu\left(F_{n}^{*} \cap F_{n^{\prime}}^{*}\right)-\mu\left(F_{n}^{*}\right) \mu\left(F_{n^{\prime}}^{*}\right)\right) \\
& \leq \sum_{n=N_{1}}^{N_{2}} \sum_{n^{\prime}=N_{1}}^{n-1}\left(\sum_{k=n-n^{\prime}}^{n} \mu\left(F_{n, k}^{*} \cap F_{n^{\prime}, n^{\prime}+k-n}^{*}\right)\right) \leq \sum_{n=N_{1}}^{N_{2}} \sum_{n^{\prime}=N_{1}}^{n-1}\left(\sum_{k=n-n^{\prime}}^{n} \mu\left(F_{n, k}^{*}\right)\right) .
\end{aligned}
$$

Since $\mu\left(F_{n, k}^{*}\right)=\frac{q-1}{q} \cdot \frac{1}{q^{\frac{1}{l_{n}+2 k}}}$, we have

$$
\begin{aligned}
\sum_{n=N_{1}}^{N_{2}} \sum_{n^{\prime}=N_{1}}^{n-1}\left(\mu\left(F_{n}^{*} \cap F_{n^{\prime}}^{*}\right)-\mu\left(F_{n}^{*}\right) \mu\left(F_{n^{\prime}}^{*}\right)\right) & \ll \sum_{n=N_{1}}^{N_{2}} \sum_{n^{\prime}=N_{1}}^{n-1} \frac{1}{q^{l_{n}}} \cdot \frac{1}{q^{n-n^{\prime}}} \\
& \ll \sum_{n=N_{1}}^{N_{2}} \frac{1}{q^{l_{n}}} \ll \sum_{n=N_{1}}^{N_{2}} \mu\left(F_{n}^{*}\right) .
\end{aligned}
$$


Thus from (6) and (7) we have (3). Now we estimate $\left|\widetilde{\Phi}(N)-\frac{q}{q+1} \Phi(N)\right|$. From Lemma 2, we have

$$
\mu\left(F_{n}^{*}\right)=\sharp W_{n}^{*} \cdot \frac{1}{q^{l_{n}+2 n}}=\frac{q}{q+1} \cdot \frac{1}{q^{l_{n}}}+\frac{1}{q+1} \cdot \frac{1}{q^{2 n+l_{n}}} .
$$

Thus we see that

$$
\begin{aligned}
\left|\widetilde{\Phi}(N)-\frac{q}{q+1} \Phi(N)\right| & \\
& =\left|\sum_{n=1}^{N}\left(\frac{q}{q+1} \cdot \frac{1}{q^{l_{n}}}+\frac{1}{q+1} \cdot \frac{1}{q^{2 n+l_{n}}}\right)-\frac{q}{q+1} \sum_{n=1}^{N} \frac{1}{q^{l_{n}}}\right| \\
& =\frac{1}{q+1} \sum_{n=1}^{N} \frac{1}{q^{2 n+l_{n}}}<1 .
\end{aligned}
$$

This implies the assertion of the theorem.

3. The number of solutions as pairs of polynomials. We consider solutions $(P, Q) \in W_{n}^{* *}$ of

$$
\left|f-\frac{P}{Q}\right|<\frac{1}{q^{2 n+l_{n}}}
$$

for $f \in \mathbb{L}$. As mentioned in the introduction, the number of solutions of

$$
\left|f-\frac{P}{Q}\right|<\frac{1}{q^{2 n+l_{n}}}, \quad \operatorname{deg} Q=n,(P, Q)=1,1 \leq n \leq N,
$$

is $\frac{q-1}{q} \Phi(N)+O\left(\Phi(N)^{1 / 2} \log ^{3 / 2+\varepsilon} \Phi(N)\right)$. Suppose that $(P, Q)=(R \widehat{P}, R \widehat{Q})$ for $R \in \mathbb{F}_{q}[X]$ with $\operatorname{deg} R=k$ and co-prime polynomials $\widehat{P}, \widehat{Q}$. Then

$$
\left|f-\frac{P}{Q}\right|<\frac{1}{q^{2 n+l_{n}}} \quad \text { implies } \quad\left|f-\frac{\widehat{P}}{\widehat{Q}}\right|<\frac{1}{q^{2(n-k)+l_{n}+2 k}} .
$$

On the other hand, if we have such a $\widehat{P} / \widehat{Q}$, then there are $(q-1) q^{k}$ choices of $R$ with $\operatorname{deg} R=k$, i.e. $(q-1) q^{k}$ solutions $(P, Q)$ arising from $(\widehat{P}, \widehat{Q})$. Thus we have the following.

Theorem 2. For $\mu$-a.e. $f$, we have

$$
\liminf _{N \rightarrow \infty} \frac{\sharp\left\{(P, Q) \in W_{n}^{* *}: 1 \leq n \leq N,(8) \text { holds }\right\}}{\Phi(N)} \geq q-1 .
$$


Proof. The number of solutions of (8) with $1 \leq n \leq N$ is equal to

$$
\begin{aligned}
(q-1) \sharp\left\{\frac{P}{Q}\right. & \left.\in W_{n}:\left|f-\frac{P}{Q}\right|<\frac{1}{q^{2 n+l_{n}}}\right\} \\
& +(q-1) q \sharp\left\{\frac{P}{Q} \in W_{n-1}:\left|f-\frac{P}{Q}\right|<\frac{1}{\left.q^{2(n-1)+\left(l_{n}+2\right)}\right\}}\right. \\
& +(q-1) q^{2} \sharp\left\{\frac{P}{Q} \in W_{n-2}:\left|f-\frac{P}{Q}\right|<\frac{1}{q^{2(n-2)+\left(l_{n}+4\right)}}\right\}+\cdots \\
& +(q-1) q^{n-1} \sharp\left\{\frac{P}{Q} \in W_{1}:\left|f-\frac{P}{Q}\right|<\frac{1}{q^{2+\left(l_{n}+2(n-1)\right)}}\right\} \\
& \left(+(q-1) q^{n} \quad \text { if }|f|<\frac{1}{q^{2 n+l_{n}}}\right) .
\end{aligned}
$$

Obviously, $|f|>1 / q^{2 n+l_{n}}$ for sufficiently large $n$ unless $f \equiv 0$. We fix a positive integer $K$ and suppose $K<N$. For any $0 \leq k \leq K$ we have

$$
\begin{aligned}
\sharp\left\{\frac{P}{Q} \in W_{n}\right. & \left.: 1 \leq n \leq N-k,\left|f-\frac{P}{Q}\right|<\frac{1}{q^{2 n+l_{n}+2 k}}\right\} \\
& =\frac{q-1}{q} \frac{1}{q^{2 k}} \Phi(N)+O\left(\Phi(N)^{1 / 2} \log ^{3 / 2+\varepsilon} \Phi(N)\right) \quad \text { for } \mu \text {-a.e. } f .
\end{aligned}
$$

Now

$$
\begin{aligned}
\sharp\left\{(P, Q) \in W_{n}^{* *}:\right. & 1 \leq n \leq N,(8) \text { holds }\} \\
& \geq \sum_{k=0}^{K}(q-1) q^{k} \sharp\left\{\frac{P}{Q} \in W_{n-k}:\left|f-\frac{P}{Q}\right|<\frac{1}{q^{2(n-k)+l_{n}+2 k}}\right\}
\end{aligned}
$$

and the right hand side is

$$
\sim \sum_{k=0}^{K}(q-1) q^{k} \frac{q-1}{q} \frac{1}{q^{2 k}} \Phi(N)=(q-1)\left(1-\frac{1}{q^{K+1}}\right) \Phi(N) \quad \text { for } \mu \text {-a.e. } f \text {. }
$$

This implies the assertion of the theorem.

To bound the limsup, we need some conditions on $\left(l_{n}: n \geq 1\right)$. Before we discuss that, we recall some facts on continued fractions of formal Laurent power series.

For any $f \in \mathbb{L}$ there exists a unique continued fraction expansion of the following form:

$$
f=\frac{1}{A_{1}+\frac{1}{A_{2}+\ddots}}, \quad A_{n} \in \mathbb{F}_{q}[X] \backslash\{0\} .
$$


This expansion is finite if and only if $f \in \mathbb{F}_{q}(X)$. As usual, we put

$$
\left\{\begin{array} { l } 
{ P _ { 0 } = 0 , P _ { 1 } = 1 , } \\
{ Q _ { 0 } = 1 , Q _ { 1 } = A _ { 1 } , }
\end{array} \text { and } \left\{\begin{array}{l}
P_{n+1}=A_{n+1} P_{n}+P_{n-1}, \\
Q_{n+1}=A_{n+1} Q_{n}+Q_{n-1},
\end{array}\right.\right.
$$

and have the $n$th convergent $P_{n} / Q_{n}$ for $n \geq 0$. The following are well known (e.g. V. Berthé and H. Nakada [1]):

$$
\left|f-\frac{P}{Q}\right|<\frac{1}{|Q|^{2}} \text { with } Q \neq 0 \text { if and only if } \frac{P}{Q}=\frac{P_{n}}{Q_{n}} \text { for some } n \geq 0,
$$

$$
\left|f-\frac{P_{n}}{Q_{n}}\right|=\frac{1}{\left|Q_{n}\right|\left|Q_{n+1}\right|}=\frac{1}{\left|Q_{n}\right|^{2}\left|A_{n+1}\right|},
$$

where $\left(A_{n}\right)$ is an independent and identically distributed sequence of $\mathbb{F}_{q}[X]$ valued random variables with respect to $\mu$, and

$$
\lim _{N \rightarrow \infty} \frac{1}{N} \operatorname{deg} Q_{N}=\lim _{N \rightarrow \infty} \frac{1}{N} \sum_{n=1}^{N} \operatorname{deg} A_{n}=\frac{q}{q-1} \quad \text { for } \mu \text {-a.e. } f \text {. }
$$

Now we assume the conditions (A1) and (A2) stated in $\S 1$. Then we have the following.

ThEOREM 3. Under the conditions (A1) and (A2), we have

$$
\lim _{N \rightarrow \infty} \frac{\sharp\left\{(P, Q) \in W_{n}^{* *}: 1 \leq n \leq N,(8) h o l d s\right\}}{\Phi(N)}=q-1 \quad \text { for } \mu \text {-a.e. } f \text {. }
$$

Proof. If $\left(l_{n}: n \geq 1\right)$ is constant for sufficiently large $n$, the statement of the theorem follows immediately from (9)-(11). So we assume that $l_{n} \rightarrow \infty$. To prove the assertion, we only need to show that for $\mu$-a.e. $f$,

$$
\sharp\left\{(P, Q) \in W_{n}^{* *}: 1 \leq n \leq N,(8) \text { holds }\right\} \leq(q-1) \Phi(N)(1+\varepsilon)
$$

where $\varepsilon>0$ is arbitrary and $N$ is sufficiently large.

For this purpose, we change $\left(l_{n}: n \geq 1\right)$ to $\left(\widetilde{l}_{m}: m \geq 1\right)$ for counting the number of solutions associated to continued fraction expansions. From (9), for a given $(P, Q)$ such that

$$
\left|f-\frac{P}{Q}\right|<\frac{1}{|Q|^{2}}
$$

there exists a unique $m \geq 0$ such that

$$
\frac{P}{Q}=\frac{P_{m}}{Q_{m}} .
$$

In this case, there exist $R \in \mathbb{F}_{q}[X]$ and $t \geq 1$ such that $Q=R Q_{m}$ and

$$
\left|f-\frac{P}{Q}\right|=\frac{1}{|Q|^{2} q^{t}} \text {. }
$$


This means

$$
\left|f-\frac{P_{m}}{Q_{m}}\right|=\frac{1}{\left|Q_{m}\right|^{2} q^{t+2 \operatorname{deg} R}} .
$$

From (10) we see that

$$
t+2 \operatorname{deg} R=\operatorname{deg} A_{m+1}
$$

and so

$$
\operatorname{deg} Q<\operatorname{deg} Q_{m+1} \text {. }
$$

Suppose that $l_{n}$ is constant for $n \in\left[\operatorname{deg} Q_{m}, \operatorname{deg} Q_{m+1}\right)$. This implies the following: if $\operatorname{deg} Q_{m}=u$ and

$$
\left|f-\frac{P_{m}}{Q_{m}}\right|=\frac{1}{q^{2 u+l_{n}+2 t+1}} \text { or } \frac{1}{q^{2 u+l_{n}+2 t+2}},
$$

then there are

$$
(q-1)+(q-1) q+(q-1) q^{2}+\cdots+(q-1) q^{t}
$$

solutions $(P, Q)$ of (8) such that $\operatorname{deg} Q_{m} \leq \operatorname{deg} Q<\operatorname{deg} Q_{m+1}$. Here we note that $\operatorname{deg} A_{m+1}=l_{n}+2 t+1$ or $l_{n}+2 t+2$. Fix a sufficiently small $\varepsilon>0$. We put $z_{k}=l_{j_{k}}-l_{j_{k-1}}$ for $k \geq 2$. By the assumption (A2), $(1+\varepsilon) j_{k}<j_{k+1}$ for $k \geq 1$. So the following is well defined:

$$
\tilde{l}_{m}= \begin{cases}l_{\left[\frac{q}{q-} m\right]}-z_{k} & \text { if } j_{k} \leq \frac{q}{q-1} m<(1+\varepsilon) j_{k} \text { for some } k \geq 1, \\ l_{\left[\frac{q}{q-1} m\right]} & \text { otherwise. }\end{cases}
$$

From (11), for $\mu$-a.e. $f$ there exists $m_{0}$ such that

$$
\operatorname{deg} Q_{m} \in\left[\frac{q}{q-1} m\left(1-\frac{\varepsilon}{2}\right), \frac{q}{q-1} m\left(1+\frac{\varepsilon}{2}\right)\right) \quad \text { for } m \geq m_{0} .
$$

We choose $N_{0}$ so that

$$
\frac{q}{q-1} m_{0}\left(1+\frac{\varepsilon}{2}\right) \leq N_{0}<\frac{q}{q-1} m_{0}\left(1+\frac{\varepsilon}{2}\right)+1 .
$$

We consider a sufficiently large integer $N\left(>N_{0}\right)$ and choose an integer $m_{1}$ so that

$$
N \leq \frac{q}{q-1} m_{1}\left(1-\frac{\varepsilon}{2}\right)<N+1 .
$$

We have the following.

LEMMA 3.

$$
\begin{aligned}
& \sharp\left\{(P, Q) \in W_{n}^{* *}:\left|f-\frac{P}{Q}\right|\right.\left.<\frac{1}{q^{2 n+l_{n}+v}},(P, Q)=1, N_{0}<n \leq N\right\} \\
& \leq \sharp\left\{m_{0} \leq m<m_{1}:\left|f-\frac{P_{m}}{Q_{m}}\right|<\frac{1}{\left|Q_{m}\right|^{2} q^{\widetilde{l}_{m}+v}}\right\}
\end{aligned}
$$

for any $v \geq 1$. 
Proof. Suppose

$$
\left|f-\frac{P}{Q}\right|<\frac{1}{q^{2 n+l_{n}+v}}
$$

with $N_{0}<\operatorname{deg} Q \leq N$ and $(P, Q)=1$. There exists $m \geq 1$ such that $P / Q=P_{m} / Q_{m}$. Since

$$
\operatorname{deg} Q_{m_{0}}<\frac{q}{q-1} m_{0}\left(1+\frac{\varepsilon}{2}\right) \leq N_{0}<N \leq \frac{q}{q-1} m_{1}\left(1-\frac{\varepsilon}{2}\right) \leq \operatorname{deg} Q_{m_{1}},
$$

we see that $m_{0} \leq m \leq m_{1}$. Moreover $\widetilde{l}_{m} \leq l_{n}$ for such $m$ and $n$. Thus we have the assertion of the lemma.

Now we pick $m \geq 1$ such that $\operatorname{deg} A_{m+1}>\widetilde{l}_{m}$. We put

$$
\tau_{n}(f):=\min \left\{m>\tau_{n-1}(f): \widetilde{l}_{m}<\operatorname{deg} A_{m+1}\right\}
$$

with

$$
\tau_{1}(f):=\min \left\{m \geq 1: \widetilde{l}_{m}<\operatorname{deg} A_{m+1}\right\}
$$

We also put

$$
\psi_{n}(f):=q^{\left\lceil\left(\operatorname{deg} A_{\tau_{n}(f)+1}-\widetilde{l}_{\tau_{n}(f)}\right) / 2\right\rceil}-1,
$$

where $\lceil z\rceil$ denotes the smallest integer which is not less than $z$. Because of (13), $\psi_{n}(f)$ is the number of solutions of (8) with $\operatorname{deg} Q_{m} \leq \operatorname{deg} Q<$ $\operatorname{deg} Q_{m+1}$ for some $m \geq 1$. If we put

$$
\widetilde{n}:=\max \left\{n: \tau_{n}(f) \leq m_{1}\right\},
$$

then from Lemma 3 we have

$$
\sharp\left\{(P, Q) \in W_{n}^{* *}:\left|f-\frac{P}{Q}\right|=\frac{1}{q^{2 n+l_{n}}}, N_{0}<n \leq N\right\} \leq \sum_{n=1}^{\widetilde{n}} \psi_{n}(f) .
$$

LEMma 4.

$$
\sum_{n=1}^{K} \psi_{n}(f) \sim K q \quad \text { for } \mu \text {-a.e. } f .
$$

Proof. Since ( $\operatorname{deg} A_{m}: m \geq 1$ ) is i.i.d., (11) holds, and the distribution is $\left(\frac{q-1}{q}, \frac{q-1}{q^{2}}, \ldots\right)$, it follows that the selected sequence $\left(\operatorname{deg} A_{\tau_{n}(f)+1}-\widetilde{l}_{\tau_{n}(f)}\right.$ : $n \geq 1)$ is also i.i.d. with the same distribution. Thus it is easy to see that $E\left(\psi_{n}\right)=q$ and the strong law of large numbers implies the assertion.

Lemma 5. For each integer $K$ and $\widehat{m}$ with $\tau_{K}(f) \leq \widehat{m}<\tau_{K+1}(f)$, we have

$$
K \sim \sum_{m=1}^{\widehat{m}} \frac{1}{q^{\widetilde{l}_{m}}} \quad \text { for } \mu \text {-a.e. } f .
$$


Proof. From the definition of $\tau_{n}(f)$,

$$
K=\sharp\left\{1 \leq m \leq \widehat{m}: \operatorname{deg} A_{m+1}>\widetilde{l}_{m}\right\} .
$$

Here, $\left(\left\{f: \operatorname{deg} A_{m+1}>\widetilde{l}_{m}\right\}: m \geq 1\right)$ is a sequence of independent events and we have

$$
K \sim \sum_{m=1}^{\widehat{m}} \mu\left(\left\{f: \operatorname{deg} A_{m+1}>\widetilde{l}_{m}\right\}\right)
$$

from the Basic Lemma. Since $\mu\left(\left\{f: \operatorname{deg} A_{m+1}>\widetilde{l}_{m}\right\}\right)=1 / \widetilde{q}^{\widetilde{l}_{m}}$, we get the assertion of the lemma.

From these lemmas, we have

$$
\sum_{n=1}^{\widetilde{n}} \psi_{n}(f) \sim q \sum_{m=1}^{m_{1}} \frac{1}{q^{\widetilde{l}_{m}}} \quad \text { for } \mu \text {-a.e. } f \text {. }
$$

LEMma 6. We have

$$
\sum_{m=1}^{m_{1}} \frac{1}{\widetilde{q}^{\widetilde{l}_{m}}} \leq \frac{q-1}{q} \sum_{n=1}^{\widetilde{N}} \frac{1}{q^{l_{n}}}(1+\varepsilon)
$$

where $\widetilde{N}=\left[\frac{N+1}{1-\varepsilon / 2}\right]$ for sufficiently large $N$ with

$$
N \leq \frac{q}{q-1} m_{1}\left(1-\frac{\varepsilon}{2}\right)<N+1
$$

Proof. From the definition of $\left(\widetilde{l}_{m}: m \geq 1\right)$, we have

$$
\sum_{m=1}^{m_{1}} \frac{1}{q^{\widetilde{l}_{m}}} \sim \frac{q-1}{q}\left\{j_{1} \frac{1}{q^{l_{1}}}+j_{1} \varepsilon \frac{1}{q^{l_{j_{1}}}}+\left(j_{2}-j_{1}-j_{1} \varepsilon\right) \frac{1}{q^{l_{2}}}+j_{2} \varepsilon \frac{1}{q^{l_{j_{2}}}}+\cdots\right\} .
$$

Thus

$$
\begin{aligned}
& \sum_{m=1}^{m_{1}} \frac{1}{q^{\widetilde{l}_{m}}}-\frac{q-1}{q} \sum_{n=1}^{\widetilde{N}} \frac{1}{q^{l_{n}}} \\
& \sim \frac{q-1}{q}\left[j_{1} \varepsilon\left(\frac{1}{q^{l_{j_{1}}}}-\frac{1}{q^{l_{j_{2}}}}\right)+j_{2} \varepsilon\left(\frac{1}{q^{l_{j_{2}}}}-\frac{1}{q^{l_{j_{3}}}}\right)+\cdots+j_{\widehat{k}} \varepsilon\left(\frac{1}{q^{l_{\widehat{\jmath}}}}-\frac{1}{q^{l_{\widehat{k}+1}}}\right)\right],
\end{aligned}
$$

where $\widehat{k}$ is chosen so that $j_{\widehat{k}} \leq \widetilde{N}<j_{\widehat{k}+1}$. The right hand side is equal to

$$
\begin{aligned}
\frac{q-1}{q}\left[j_{1} \varepsilon \frac{1}{q^{l_{j_{1}}}}+\left(j_{2}-j_{1}\right) \varepsilon \frac{1}{q^{l_{j_{2}}}}+\cdots+\left(j_{\widehat{k}}-j_{\widehat{k}-1}\right) \varepsilon \frac{1}{q^{l_{j_{\widehat{k}}}}}\right. & \left.-j_{\widehat{k}} \varepsilon \frac{1}{q^{l_{\widehat{k}+1}}}\right] \\
& \leq \frac{q-1}{q} \varepsilon \sum_{n=1}^{\widetilde{N}} \frac{1}{q^{l_{n}}},
\end{aligned}
$$

which completes the proof of the lemma. 
Since $\widetilde{N} \leq N(1+\varepsilon)$ and $\left(l_{n}: n \geq 1\right)$ is non-decreasing, we see that

$$
\begin{aligned}
\sum_{n=1}^{\widetilde{N}} \frac{1}{q^{l_{n}}} & =\sum_{n=1}^{N} \frac{1}{q^{l_{n}}}+\sum_{n=N+1}^{\tilde{N}} \frac{1}{q^{l_{n}}} \leq \sum_{n=1}^{N} \frac{1}{q^{l_{n}}}+\sum_{n=N+1}^{[N(1+\varepsilon)]} \frac{1}{q^{l_{n}}} \\
& \leq \sum_{n=1}^{N} \frac{1}{q^{l_{n}}}(1+\varepsilon)=\Phi(N)(1+\varepsilon) .
\end{aligned}
$$

Hence

$$
\sum_{m=1}^{m_{1}} \frac{1}{q^{\widetilde{l}_{m}}} \leq \frac{q-1}{q} \sum_{n=1}^{N} \frac{1}{q^{l_{n}}}(1+\varepsilon)^{2}=\frac{q-1}{q} \Phi(N)(1+\varepsilon)^{2} .
$$

Consequently from (14), (15), and Lemma 6, we have (12), which implies the assertion of the theorem.

\section{References}

[1] V. Berthé and H. Nakada, On continued fraction expansions in positive characteristic: equivalence relations and some metric properties, Expo. Math. 18 (2000), 257-284.

[2] K. Inoue and H. Nakada, On metric Diophantine approximation in positive characteristic, Acta Arith. 110 (2003), 205-218.

[3] G. Larcher and H. Niederreiter, Kronecker-type sequences and nonarchimedean diophantine approximations, ibid. 63 (1993), 379-396.

[4] -, 一, Generalized $(t, s)$-sequences, Kronecker-type sequences, and Diophantine approximations of formal Laurent series, Trans. Amer. Math. Soc. 347 (1995), 20512073.

[5] W. Philipp, Some metrical theorems in number theory, Pacific J. Math. 20 (1967), 109-127.

[6] V. G. Sprindžuk, Metric Theory of Diophantine Approximations, Wiley, New York, 1979 .

Hitoshi Nakada

Department of Mathematics

Keio University

3-14-1 Hiyoshi, Kohoku-ku

Yokohama 223-8522, Japan

E-mail: nakada@math.keio.ac.jp
Rie Natsui

Department of Mathematics

Keio University

3-14-1 Hiyoshi, Kohoku-ku

Yokohama 223-8522, Japan

and

Department of Mathematics

Nihon University

Sakurajosui, Setagaya-ku

Tokyo 156-8500, Japan

E-mail: r_natui@math.keio.ac.jp

Received on 23.5.2005

and in revised form on 9.2.2006 